\title{
Combined approach for Port-a-Cath implantation -a dependable alternative
}

\author{
Guruprasad Rai D ${ }^{1}$, Vijaya Kumara ${ }^{1 *}$, Arvind Kumar Bishnoi ${ }^{2}$, Ganesh S Kamath ${ }^{2}$, Rajkamal Vishnu ${ }^{3}$, \\ Harshil Joshi ${ }^{3}$, Lokavendra Singh Budania ${ }^{1}$, Yogesh K Gaude ${ }^{l}$ \\ Associate Professor ${ }^{1}$, Professor ${ }^{2}$, Assistant Professor ${ }^{3}$, Kasturba Medical College, Manipal Academy of \\ Higher Education, Manipal, India.
}

\begin{abstract}
Background: Port-a-Cath are routinely warranted as a universal venous access for chemotherapy, due to their operational ease, comparatively less complications and relative ease of maintenance. Catheter implantation can be done either by surgical approach by vein cut-down technique or ultrasound-guided percutaneous technique or by landmark technique using fluoroscopy. Here we present our data on our novel technique.
\end{abstract}

Methods: A descriptive cross-sectional study of 92 consecutive patients who underwent Port-aCath implantation for adjuvant therapy, from August 2017 through to July 2019 was done. The procedure was carried out in the minor operation theatre under local anaesthesia with anaesthetist doing a transthoracic echocardiography and surgeon doing a cephalic vein cut down under echocardiography guidance, as a day care procedure. This allowed the catheter to reach the superior vena cava smoothly thus avoiding inadvertent cannulation of the internal jugular vein.

Results: Four (4.34\%) of 92 patients required additional sedation. No patients required to be reexplored in lieu of erroneous placement of the catheter. In all patients the catheter was placed properly in the superior vena cava- right atrial junction. Late complications amounted to $8.69 \%$ of the study group, this included flip over, puncture site infection and catheter blockage. All patients were followed-up for a period of 1 to 6 months.

Conclusions: We advocate a combined approach of echocardiography guided Port-a-Cath insertion through cephalic vein cut down as a simple, safe, with satisfactory success rate. It offers a suitable alternative to the other invasive procedures performed under image guidance and avoids radiation exposure and malposition.

Keywords: Cephalic vein; Port-a-Cath; chemotherapy; echocardiography; vein cut down

\section{Introduction}

The implantable subcutaneous venous access port (Port-a-Cath) is routinely used for delivery of chemotherapeutic agents in cancer patients requiring long-term venous access due to its operational ease. ${ }^{1,2}$ Port-a-Cath placement is performed either with subclavian or external jugular vein puncture, by Seldinger approach

\footnotetext{
*Correspondence: Vijaya Kumara

E mail: vijaysd84@gmail.com

iD

https://orcid.org/0000-0003-4642-7213

Received: 09/04/2020

Accepted: 13/06/2020

DOI: http:/doi.org/10.4038/slja.v28i2.8570
}

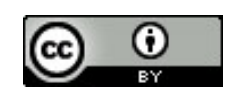

(landmark technique or under ultrasound guidance); or surgical approach by venous cutdown. ${ }^{3}$ Many randomized control trials have recommended that using ultrasound (US) guidance decreases complication rates and increases firstpass success, making it the standard of care when performing a central venous catheter placement., National Institute for Clinical Excellence (NICE) guidelines has also suggested that ultrasound guidance should be used for central venous catheter (CVC) placement. $^{6}$ The peri procedural complication cited with percutaneous ultrasound guided techniques are arterial puncture, brachial plexus injuries, deep venous thrombosis, and pneumothorax. ${ }^{7,8}$ Surgical approach with peripheral vein cut-down technique (cephalic vein) is used only infrequently. Although this technique has been described, very few prospective data are available on the cephalic vein cut down technique. ${ }^{9}$ Recent 
literature have shown similar success rate, as well as complication rates, when comparing percutaneous vein puncture and surgical approach. ${ }^{7}$

For catheter implantation, image-guided venous puncture, using fluoroscopy is considered the safest option as malposition is completely avoided. ${ }^{10,11}$ Fluoroscopy use exposes the patient and healthcare personnel to ionizing radiation that can have deleterious health effects in the long term. We must continue to strive to minimize radiation burden but without compromising diagnostic accuracy or procedural safety. Guidelines favour ultrasound guided percutaneous approach over surgical cut down, but both the approaches need a post procedure x-ray or fluoroscopy for confirmation of appropriate placement of the catheter tip. ${ }^{12}$ Either surgical cut down or percutaneous techniques have their own quandary so this lead us to introduce a surrogate approach of venous cut down combined with echocardiography. The objective of our study was to analyze feasibility and outcomes of novel technique performed under echo guidance.

\section{Methodology}

A descriptive cross-sectional study of 92 consecutive patients who underwent Port-a-Cath implantation for adjuvant therapy, from August 2017 to July 2019 was done. Institutional Research Committee and Institutional Ethical Committee clearance were taken prior to conducting the study. Implantation of the Port-a-Cath was carried out under maximal sterile barrier precautions in the minor operation theatre under monitored anaesthesia care (MAC). The location for implantation was chosen to be the right infraclavicular region, in all patients. The length of the incision was around three inches, two centimeters below the clavicle, such that the lateral part of the incision touched the deltopectoral groove. The incision was then deepened till the pectoralis fascia was visualized, and a subcutaneous enclave was created for the chamber. The cephalic vein was then identified in the deltopectoral groove and isolated. The length of the catheter was premeasured from the infraclavicular area up to the 2 nd intercostal space. It was introduced into the cephalic vein and advanced, with the neck turned towards the left (Figure 1,2). Point-of-care 2D echocardiography was done by the anaesthetist to identify appropriate placement of the catheter in the right atrial (RA)/superior vena cava (SVC) junction. In this technique the catheter was slowly advanced through the cephalic vein until a bright, structure was identified on transthoracic echocardiography in the right atrium in an apical four chamber view, which represents the tip of the catheter. After the tip of the catheter was located in the right atrium, the catheter was slightly pulled back until it disappeared from the right atrium. The catheter was positioned at the SVC - RA junction (Figure 3). The external end of catheter was then connected to the port which was secured to the pectoral fascia with monofilament sutures, within the subcutaneous enclave (Figure 4). Aspiration was done to check the back flow and the port was flushed with $2 \mathrm{ml}$ of heparin $(1 \mathrm{ml}=1000$ units) via a deflected point Huber's needle. Haemostasis was secured and the wound closure was done.

Figure 1: Cephalic vein dissection

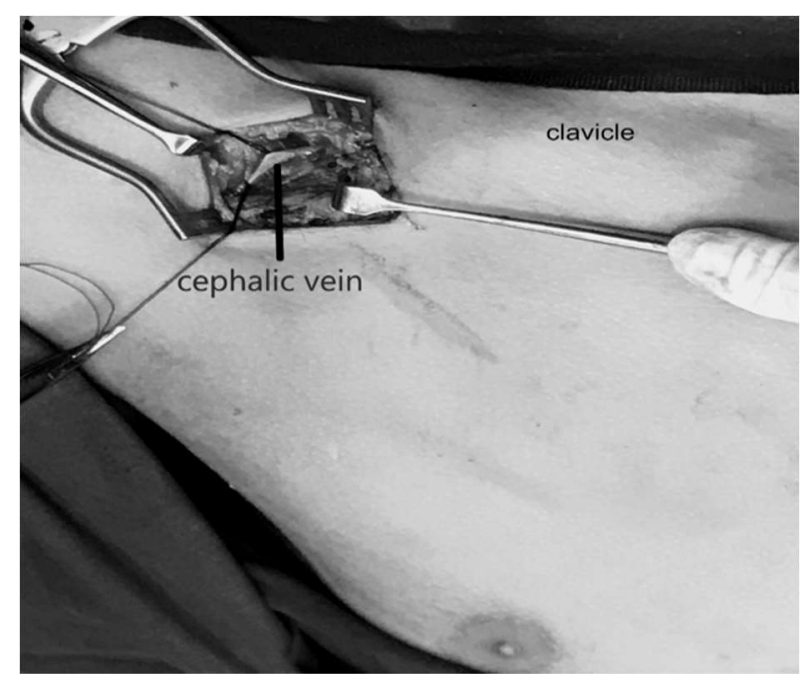

Figure 2: Catheter inserted through cephalic vein

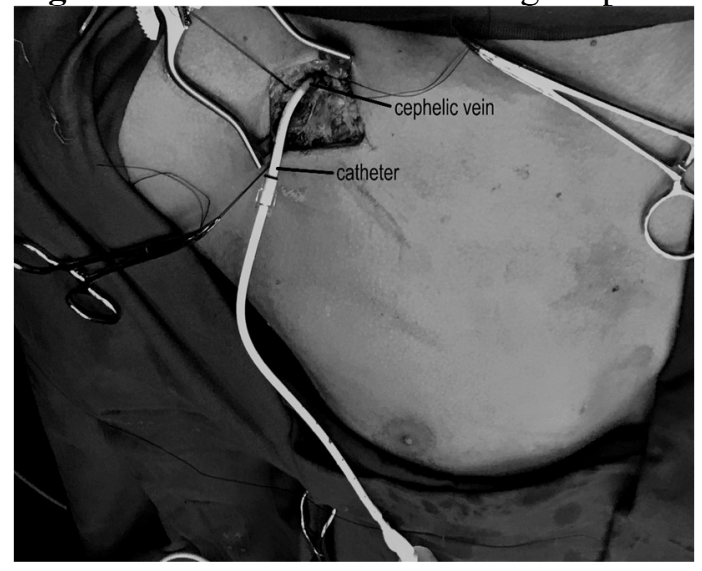


Figure 3: Four chamber view on echocardiography showing the catheter tip

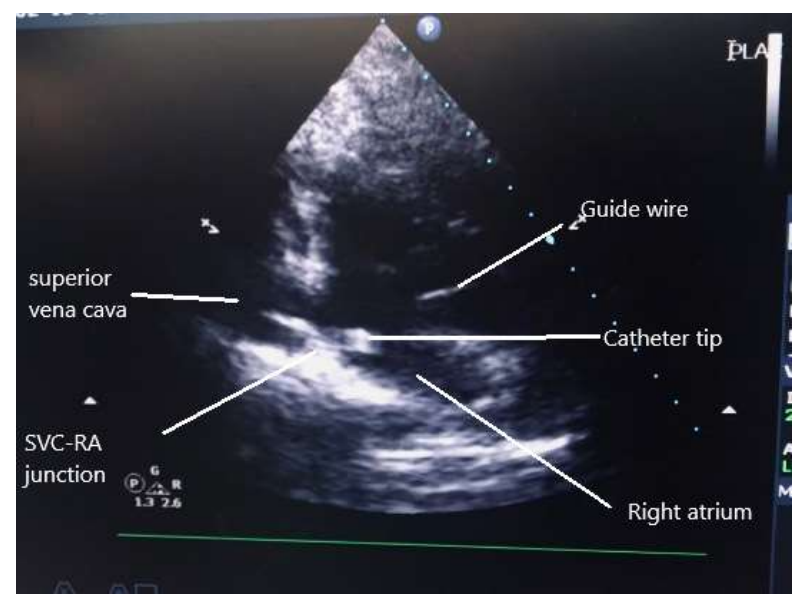

Figure 4: Chemoport connected to the catheter

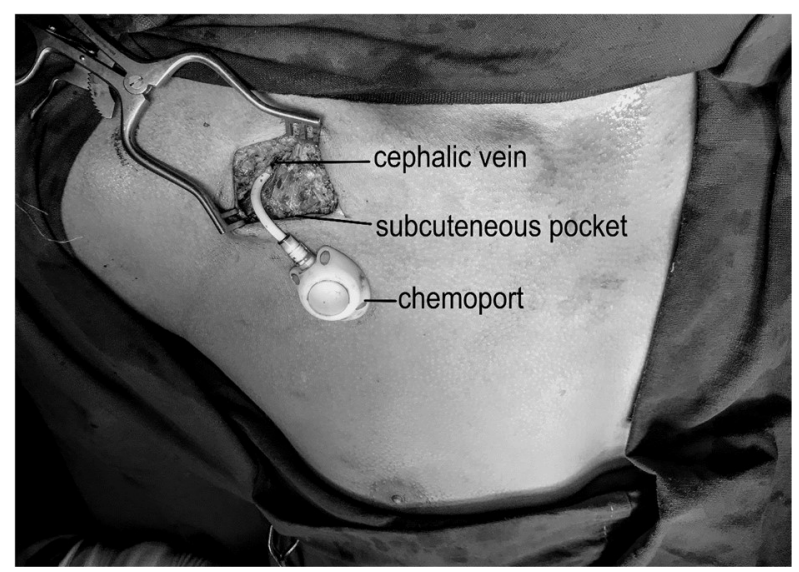

In all the cases, catheter was well positioned at the superior vena cava to right atrial junction or slightly above this level. Postoperatively, chemotherapy commenced next day after the procedure. Flushing of the port with heparinized saline was mandatory after each cycle of chemotherapy and every month thereafter.

Data analysis: data was entered and analyzed using SPSS version 15 . The results are summarized as percentages and proportions.

\section{Results}

A total of 92 patients underwent the procedure under echocardiography guidance under local anaesthesia, among these $66(71.7 \%)$ patients were males and $26(28.3 \%)$ were females with a mean age of 47 years (range 14-67 years). The various underlying malignancies included colorectal cancer
(39.1\%), breast cancer (23.9\%), nasopharyngeal cancer $(6.5 \%)$, ovarian cancer (19.5\%), lymphoma $(2.1 \%)$ and carcinoma bronchus (8.6\%) among others.

There was no scope for arterial puncture with this technique and no incidence of pneumothorax, haemothorax or brachial plexus injury following the procedure. In four patients $(4.34 \%)$, cephalic vein caliber was small and needed cannulation of the subclavian vein. These patients required additional sedation. The catheter was successfully positioned in SVC-RA junction in all the cases. As procedure is done under echocardiography guidance, we did not have any erroneous placement of the catheter. Patients were followed up for a period of 6 months post procedure. Late complications included two cases of puncture site infection due to poor hygiene $(2.17 \%), 4$ cases of catheter blockade $(4.35 \%)$ and two cases of flip over $(2.17 \%)$. We did not have any cases of central vein thrombosis.

\section{Discussion}

The Port-a-Cath is an excellent device for venous access for patients on long term chemotherapy. This system alleviates the need for multiple venipunctures, pain and risk of thrombophlebitis. Earlier traditional landmark based approach using fluoroscopy was used for placement of Port a Cath, but now it is considered obsolete. ${ }^{10,11}$ Again, any decrease in exposure to ionizing radiation should be seen as a good first step. On the contrary, the cephalic vein cut down approach has been described as a safe and useful alternative to the percutaneous subclavian vein approach by many authors. ${ }^{9,13}$ This open, cut-down of the cephalic vein done within the minor operation theatre, is a quick learnskill.

This reduces the cost of the procedure. Pneumothorax is virtually ruled out as there is no blind puncture of any vein thereby abolishing inadvertent pleural injury. The major pitfall is the inability to obtain a correct placement of the catheter which is a relatively common complication and to confirm final catheter position either intraoperative fluoroscopy or post procedural chest radiograph or both are needed. ${ }^{14}$

Recent literatures have shown shifting of standard of care when performing a central venous catheter 
(CVC) from landmark and surgical based approach toward US guidance. ${ }^{15}$ Using ultrasound (US) guidance increases first-pass success and decreases complication rates. $^{3}$ Authors have reported complications like arterial puncture, brachial plexus injuries, deep venous thrombosis, and pneumothorax. Under ultrasound guidance during the initial venepuncture, unforeseen misplacement in either an unwanted vein or in an artery can still occur. ${ }^{16}$ US guidance does not assure the provider that the catheter tip is in the vena cava; generally a post procedure $\mathrm{x}$-ray or fluoroscopy is needed for confirmation. ${ }^{17,18}$ Both surgical cut down and percutaneous techniques have their own constraints so this lead us to introduce an alternate approach of venous cut down combined with echocardiography.

It has been described in the literature that 2D Echocardiography has been used for correct placement of a transvenous pacing wire to identify the pacer lead in the right ventricle. ${ }^{19}$ We used a similar technique with cephalic vein cut down along with echocardiographic guidance and focusing on the SVC/RA junction to observe the adequate placement of the catheter thus eliminating chances of misplacement. Transthoracic echocardiography can completely avoid misplacement, helps in better positioning of the line in the superior vena cava (SVC) - right atrial (RA) junction and the need for chest radiography for confirmation of adequate line placement is averted.

Mansfield et al has strongly associated the rates of failure and complications to the number of needle passes. They have reported that complication rates rose from $4.3 \%$ with one pass to $24 \%$ with more than two passes. ${ }^{20}$ Supplementary studies have found using the subclavian vein in the Seldinger technique there is a higher risk of catheter- related complications. ${ }^{15}$ In other techniques due to repeated puncture of the vein it may cause endothelial injury thus causing thrombus formation. The advantage of our technique is that it decreases the risk for thrombus formation secondary to endothelial tissue damage during the procedure as there is minimal injury to the endothelium. There were no instances of superficial thrombophlebitis of the cephalic vein.

This procedure, however reliable, has its own fallacies. The procedure is technically difficult in patients with a small-caliber cephalic vein or venous thrombosis. Yet another surgical dilemma is, inability to locate the cephalic vein, or failure to successfully cannulate the vein. In such a scenario, one may be required to denude the subclavian vein, deep below the clavicle. Local anaesthesia is often insufficient in such patients. This subset of patients would require additional sedation since the subclavian vein would be targeted for catheter insertion.

In our study, all the patients had appropriate catheter placement. This also reiterates that the open method with echocardiography guidance is a viable option in any subsets of patients or when imaging modalities are unavailable or when there is a financial constraint. Delayed complications such as wound infection, skin necrosis, catheter blockade is lower than other methods. ${ }^{7}$ In our study, the success rates were $100 \%$, and the complication rates were $8.69 \%$. Hence, we strongly recommend this approach as an alternative for long term venous access in terminally ill patients.

Our study is probably the first one with respect to this technique and hence has its limitations in being a descriptive study design and no prior studies to compare with. We also acknowledge that the small sample size may not give adequate statistical strength to the study and hence would limit the extrapolation of the results from the study to the general population.

\section{Conclusions}

Experience from our institute suggests that implantation of a Port-a-Cath through the cephalic vein cut down approach is simple quick learn skill and has reliable success rate. Echocardiography guidance increases the percentage of appropriate catheter placement. It also renders this method to be easy to adopt and it offers an added advantage of reduced exposure to radiation and lowers cost to the patient. All the recommendations given are for central venous line placement and port a Cath placement have been excluded from these guidelines. We recommend future guidelines to include Port a Cath insertion technique. A larger case series and a comparative prospective study would help to alleviate any doubts and reinstate this simple and effective procedure as a standard of care. 


\section{Acknowledgements}

We thank the Medical Records Department at Kasturba Hospital for providing permission to use case records.

\section{References}

1. Kulkarni S, Wu O, Kasthuri R, Moss JG. Centrally inserted external catheters and totally implantable ports for the delivery of chemotherapy: a systematic review and meta-analysis of device202 related complications. Cardiovascular and interventional radiology.2014;37(4):990-1008.

https://doi.org/10.1007/s00270-013-0771-3

PMid:24218174

2. Schwarz RE, Groeger JS, Coit DG. Subcutaneously implanted central venous access devices in cancer patients: a prospective analysis. Cancer.

1997;79(8):1635-40.

https://doi.org/10.1002/(SICI)1097-

0142(19970415)79:8<1635::AID-

CNCR30>3.0.CO;2-X

3. Brooks AJ, Alfredson M, Pettigrew B, Morris DL. Ultrasound-guided insertion of subclavian venous access ports. Annals of the Royal College of Surgeons of England. 2005; 87(1):25.

https://doi.org/10.1308/1478708051441

PMid:15720903 PMCid:PMC1963836

4. Milling TJ, Rose J, Briggs WM, et al Randomized, Controlled clinical trial of point-of-care limited ultrasonography assistance of central venous cannulation: the Third Sonography Outcomes Assessment Program (SOAP-3) Trial. Critical care medicine. 2005; 33(8):1764-9.

https://doi.org/10.1097/01.CCM.0000171533.9285 6.E5

PMid:16096454

5. Dodge KL, Lynch CA, Moore CL, et al. Use of ultrasound guidance improves central venous catheter insertion success rates among junior residents. Journal of Ultrasound in Medicine. 2012; 31(10):1519-26.

https://doi.org/10.7863/jum.2012.31.10.1519

PMid:23011614

6. National Institute for Clinical Excellence. Guidance on the use of ultrasound locating devices for placing central venous catheters. http ://www.nice.org.uk $/$ nicemedia/pdf/Ultrasound _49_GUIDANCE. Pdf. 2002.

7. Cavallaro G, Sanguinetti A, Iorio O, et al. Ultrasound-Guided Vein Puncture Versus Surgical Cut-Down Technique in Totally Implantable Venous Access Devices (Tivads): A Prospective Comparative Study on Safety, Efficacy and Complications. International surgery. 2014; 99(4):475-8.
https://doi.org/10.9738/INTSURG-D-14-00008.1

PMid:25058787 PMCid:PMC4114383

8. Di Carlo I, Pulvirenti E, Mannino M, Toro A. Increased use of percutaneous technique for totally implantable venous access devices. Is it real progress? A 27-year comprehensive review on early complications. Annals of surgical oncology. 2010; 17(6):1649-56.

https://doi.org/10.1245/s10434-010-1005-4

PMid:20204533

9. Chang HM, Hsieh HF, Hsu SD, et al. Guidewire assisted cephalic vein cut down for insertion of totally implantable access ports. Journal of surgical oncology. 2007; 95(2):156-7.

https://doi.org/10.1002/jso.20584

PMid:17262733

10. Teichgräber UK, Kausche S, Nagel SN, Gebauer B. Outcome analysis in 3,160 implantations of radiologically guided placements of totally implantable central venous port systems. European Radiology. 2011; 21(6):1224-32.

11. Funaki B, Szymski GX, Hackworth CA, et al. Radiologic placement of subcutaneous infusion chest ports for long-term central venous access. AJR. American journal of roentgenology. 1997; 169(5):1431-4. https://doi.org/10.2214/ajr.169.5.9353475 PMid:9353475

12. Wolosker N, Yazbek G, Nishinari K, et al. Totally implantable venous catheters for chemotherapy: experience in 500 patients. Sao Paulo Medical Journal. 2004; 122(4):147-51.

https://doi.org/10.1590/S1516-

31802004000400003

PMid: 15543368

13. D'Angelo FA, Ramacciato G, Aurello P, et al. Prospective randomized study of cephalic vein cutdown versus subclavian vein puncture technique in the implantation of subcutaneous venous access devices. Chirurgiaitaliana. 2002; 54(4):495-500.

14. Nocito A, Wildi S, Rufibach K, Clavien PA, Weber M. Randomized clinical trial comparing venous cutdown with the Seldinger technique for placement of implantable venous access ports. British Journal of Surgery: Incorporating European Journal of Surgery and Swiss Surgery. 2009; 96(10):1129-34.

15. Zerati AE, Wolosker N, Luccia ND, Puech-Leão P. Totally implantable venous catheters: history, implantation technique and complications. Jornal Vascular Brasileiro. 2017; 16(2):128-39. https://doi.org/10.1590/1677-5449.008216 PMid:29930637 PMCid:PMC5915861

16. Blaivas M. Video analysis of accidental arterial cannulation with dynamic ultrasound guidance for central venous access. Journal of Ultrasound in Medicine. 2009; 28(9):1239-44. 
https://doi.org/10.7863/jum.2009.28.9.1239

PMid:19710223

17. Gebauer B, El-Sheik M, Vogt M, Wagner HJ. Combined ultrasound and fluoroscopy guided port catheter implantation-high success and low complication rate. European journal of radiology. 2009; 69(3):517-22.

https://doi.org/10.1016/j.ejrad.2007.10.018 PMid:19340958

18. Rupp SM, Apfelbaum JL, Blitt C, et al. Practice guidelines for central venous access: a report by the American Society of Anesthesiologists Task Force on Central Venous Access. Anesthesiology. 2012; 116(3):539.

https://doi.org/10.1097/ALN.0b013e31823c9569

PMid:22307320

19. Bedel J, Vallée F, Mari A, et al. Guide wire localization by transthoracic echocardiography during central venous catheter insertion: a periprocedural method to evaluate catheter placement. Intensive care medicine. 2013; 39(11):1932-7.

https://doi.org/10.1007/s00134-013-3097-3 PMid:24052186

20. Mansfield PF, Hohn DC, Fornage BD, et al. Complications and failures of subclavian-vein catheterization. New England Journal of Medicine. 1994; 331(26):1735-8.

https://doi.org/10.1056/NEJM199412293312602

PMid:7984193 